\title{
Notre experience des resections etendues de l'albuginee associees aux implants peniens dans les formes severes de la maladie de La Peyronie
}

\author{
M. EtcheverRy (1), J. TignOL (2)
}

(1) Service d'Uro-Andrologie Clinique Bordeaux-Nord 15 Rue Claude Boucher 33000 Bordeaux

(2) Service Universitaire de Psychiatrie C.H. Charles Perrens 121 Rue De La Bechade 33076 Bordeaux

\section{RESUME}

Après avoir défini les formes sévères de la maladie de La Peyronie, les auteurs rapellent les principales techniques connues et proposent une simplification : recouvrement par le FASCIA DE BUCK seul, ou une membrane biologique artiflcielle résorbable.

22 observations sont analysées avec un recul de 12 à 60 mois. Les résultats montrent que la rectitude penienne est constante, et que l'allongement est de $4 \mathrm{~cm}$ en moyenne. L'érection autour des implants existe, la sensibilité du gland et 1'éjaculation réapparaissent en 4 mois en moyenne.

Mots clés : Maladie de La Peyronie; Résection des plaques ; Prothèses pénienens.

Nous entendons par formes sévères de la maladie de La Peyronie, celles qui associent :

- Coudure très accentuée, de 40 à $120^{\circ}$.

- Rétraction importante, avec raccourcicement.

- Impuissance ou impossibilité de pénétrer.

Les plicatures (tucking) ou redressements selon Nesbit ne peuvent plus être proposées, et l'on ne peut que recourir aux méthodes plus agressives, que nous rappelons :

\section{MODELAGE PENIEN SUR PROTHESE} GONFLABLE DE S.K. WILSON.

- Implantation de prothèse gonflable après forte dilatation.

- Gonflage maximal de l'implant.

- Coudure forcée dans le sens opposé à la déformation, entraînant éclatement et rupture des plaques.

- Incisions de débridement.

\section{EXCISIONS SUIVIES DE GREFFE.}

- Technique de TOM LUE.

Association d'incisions et d'excisions suivies ou non de greffes de recouvrement par des lambeaux préparés avec la veine dorsale profonde.

- Opération de KRISHNAMURTI.

Excision et couverture par greffe préputiale dermique vascularisée désépidermisée.

- Procédé d'E. AUSTONI (inspiré de DEVINE-HORTON) avec recouvrement par lambeau cutané dermique libre, après excisions très larges.

NOTRE TECHNIQUE SE VEUT PLUS $S I M P L E$, tout en étant largement inspirée des précédentes :

- Traction transbalanique pour travailler aisément. 
- Incision circulaire dans le sillon balano-préputial.

- Refoulement du fourreau jusqu'à la racine.

- Incision du fascia de Buck de chaque côté, à proximité de l'urèthre.

- Dissection et isolement sur lacs de l'ensemble Fascia de Buck-paquets vasculo-nerveux dorsaux-veine dorsale profonde, de la base du gland à la racine de la verge.

- Excision au bistouri des plaques et du septum intercaverneux à la demande. Ainsi, le pénis récupère sa souplesse, sa rectitude, et s'allonge de $4 \mathrm{~cm}$ en moyenne.

- Incision latérale de $5 \mathrm{~cm}$ à la base de chaque corps caverneux et dilatation aux bougies jusqu'au $\mathrm{N}^{\circ} 11$ habituellement, en prenant soin de ne pas perforer le tissu caverneux exposé par la résection des plaques. Ce geste n'est pas difficile, et une petite brêche n'a pas de conséquence.

- Implantation de prothèses souples $A B C$ par exemple d'un diamètre de $10 \mathrm{~mm}$ en général.

- Suture des incisions au maxon 3/0.

- Simple repose du Fascia de Buck, sans greffe de recouvrement ; on le fixe par de finssurjets de vicryl, de part et d'autre de l'urèthre.

- Suture du fourreau replacé, au sillon balanopréputial, avec ou sans circoncision selon la nouvelle situation du prépuce par rapport à l'allongement obtenu.

- Depuis 2 ans, nous essayons de compléter le recouvrement au moyen d'»UROPATCH» (® maintenant remplacé par une nouvelle membrane biologique artificielle résorbable, plus résistante (PRODURA® LABOVI).

\section{OBSERVATIONS}

22 patients ont été opérés d'avril 1992 à Mars 1996 soit un recul de 12 à 60 mois maintenant.

\section{LES LESIONS OBSERVEES}

22 fois dorsales avec prise étendue du septum, compliquées 3 fois des nodules latéraux.

\section{LA PENETRATION}

Elle était impossible chez les 22 patients, 12 fois avec impuissance totale dont 4 fois avec micro pénis acquis d'une longueur de $6 \mathrm{~cm}$ en extension, 16 fois avec angulation de 30 à $120^{\circ}$, spontanée ou provoquée par injection per opératoire sous garrot., dont 4 fois vers la droite, 1 fois ventrale, 11 fois dorsale.

\section{LA TECHNIQUE OPERATOIRE}

- Incision coronale.

- 22 fois on a pratiqué la résection septale après dissections des paquets dorsaux avec le Fascia de Buck, 4 fois une endo-cavernolyse et 1 fois une endo-cavernectomie pour fibrose totale et allongement par interposition de GORE-TEX ont dû être réalisées en complément.

\section{LES PROTHESES UTILISEES}

- Prothèses semi-rigides Dow-Corwing (Subrini) : 3 cas.

- Prothèses ABC souples - VIRILIS : 12 cas.

- Prothèses AMS 600 (Malléable) : 5 cas.

- Prothèses Dynaflex AMS (hydraulique) : 2 cas.

- Suites opératoires : ont été excellentes ; aucune complication n'a été observée.

- Aspect du pénis : a été très bon 20 fois, et 2 fois un peu moins satisfaisant.

- Gain de longueur : 20 malades avaient un raccourcissement important, on a pu allonger les verges de 2 à $6 \mathrm{~cm}$ avec une moyenne de $4 \mathrm{~cm}$.

- Reprise des raports : 17 patients ont repris entre 1 et 3 mois, soit en moyenne après 2 mois, 1 fois il a fallu 4 mois pour que la satisfaction apparaisse. 4 patients n'ont pas repris d'activité sexuelle : l'un est décédé pour artériopathie diabétique deux ans après. Deux patients ont présenté un état dépressif indépendant de notre intervention et des troubles sexuels antérieurs. 
Un patient a présenté des troubles de l'humeur à l'égard d'une épouse spécialement agressive, mais tout à fait apte à pénétrer.

- Ejaculation : elle est normalement réapparue avec les premiers rapports chez 17 patients actifs. L'orgasme est présent chez 16 patients dont un par masturbation car il n'a pas de partenaire. Il est dit diminué chez deux patients, absent dans un seul cas.

- Erection complémentaire autour des prothèses : elle peut être spontanée au cours des préliminaires ou induite au cours de la pénétration.

- Satisfaction de l'opéré :

- 13 fois totale

- 5 fois modérée

- 1 fois nulle chez un anorgasmique habituel

- 1 fois faible (patient de 76 ans)

- Satisfaction des partenaires : elle est appréciable dans 16 cas :

- 3 fois extrême

- 7 fois très bonne

- 5 fois bonne

- 1 fois insuffisante

- Sur l'angle des couples : certains sont bien satisfaits et l'expriment avec reconnaissance, même un couple pathologique dont l'homme est anorgasmique, mais dont la femme est heureuse et éprouve du plaisir après avoir fait elle même une injection intra-caverneuse, pour étoffer une érection autour des prothèses sous- jacentes. Trois le sont moins, le plaisir étant plus unilatéral et plutôt féminin.

- Les insatisfactions : proviennent de l'absence de fermeté du gland avec capotage (1 cas) et manque de désir surtout chez l'homme (3 cas).

\section{COMMENTAIRES}

Les formes sévères de la maladie de La Peyronie étant définies par l'impossibilité de pénétrations ou l'impuissance sexuelle totale, on peut dire que la récupération des possibilités de pénétration est un fait positif : il a été observé 21 fois sur 22 . Un seul pénis étant trop court pour être utilisable dans les débuts, le devient peu à peu car l'érection réapparaît et le valorise après 2 ans.

Cet élément positif est dû à la rectitude de pénis toujours récupérée et au gain de longueur moyenne de $4 \mathrm{~cm}$ qui est appréciable. Au plan opératoire, soulignons la bonne qualité des suites et l'aspect satisfaisant des organes dans la convalescence. Notons aussi une absence de morbidité et une bonne tolérance des implants.

Un point important est celui de la sensibilité distale du pénis et du gland, compte tenu des risques élevés de lésions des nerfs dorsaux au cours de la dissection. Il est important de suivre des principes techniques très rigoureux lors de la dissection du Fascia de Buck surtout vers la partie distale du pénis. Dans la majorité des cas, la sensibilité est bien conservée (18 cas), atténuée (4 cas), dont un très hypo-esthésique. La récupération est la règle en 4 mois environ. La reprise des rapports sexuels a lieu en moyenne 2 mois après l'intervention.

L'éjaculation est bien conservée sauf une fois et l'orgasme est présent chez tous ceux dont le comportement physique et psychologique est normal. Les deux sujets dépressifs restent sans désir et sans activité sexuelle, mais seraient tout à fait capables d'en avoir une. Notons comme cela a été souligné dans d'autres travaux (Jacques Brus : «Les Prothèses péniennes, à propos de 30 cas", Thèse de médecine, Bordeaux, 1983.) la présence d'érections complémentaires exactement comme dans les observations d'implantations pour d'autres étiologies.

Quant à la satisfaction, elle est plus fréquente chez les femmes que chez les patients. La reprise de la sexualité dont elles étaient privées, et la durée des rapports qui peut être allongée sont des facteurs responsables de cette donnée.

L'analyse des observations permet de faire les remarques suivantes :

- La reprise des rapports avec pénétration est 

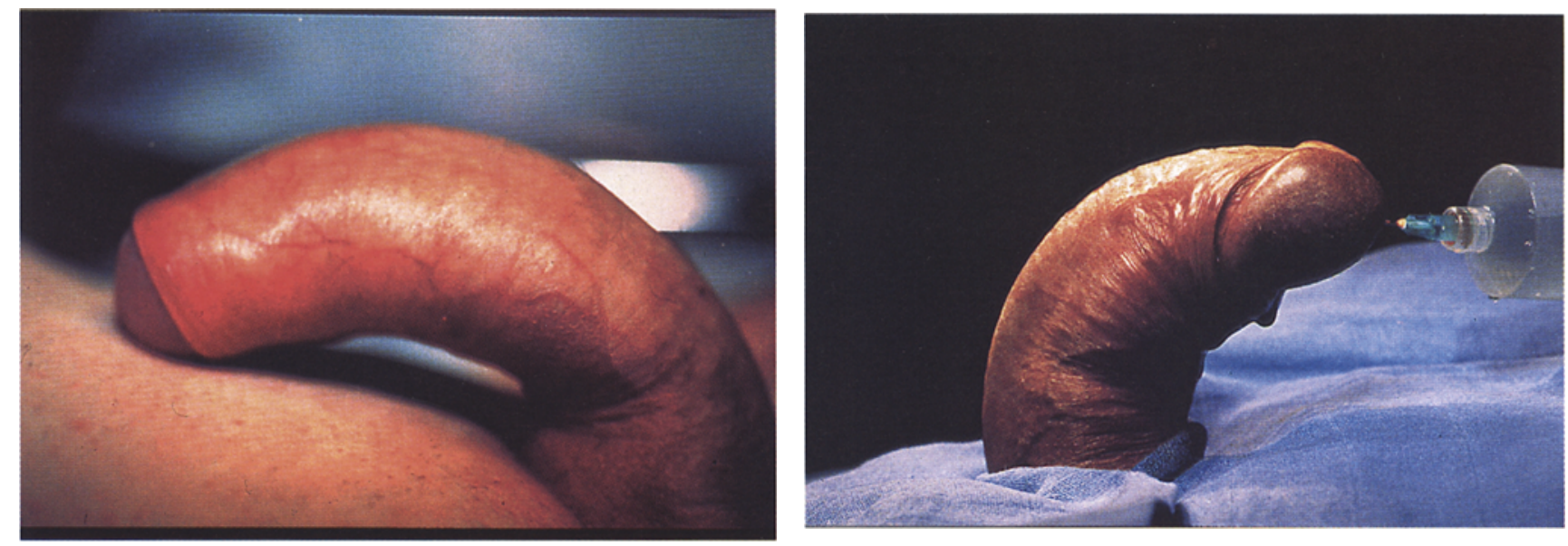

1

2

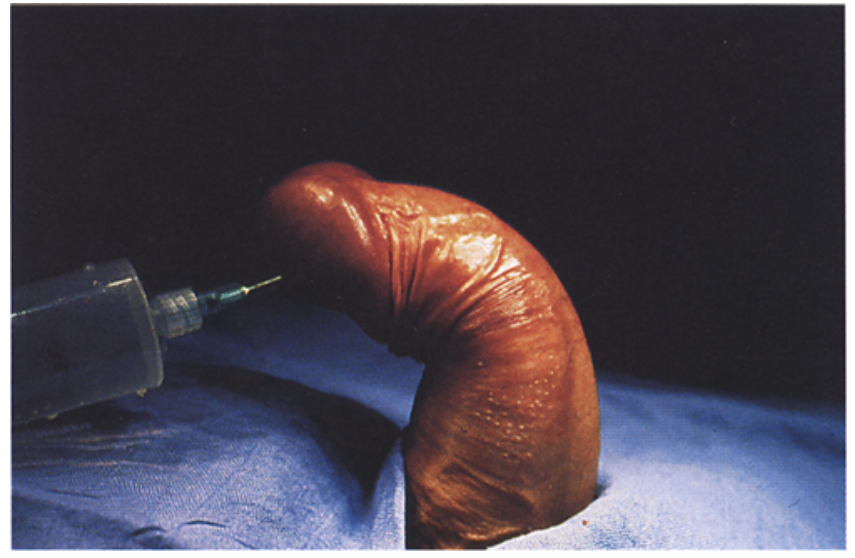

Figures 1, 2, $3: 3$ Exemples de formes sévères de maladie de la Peyronie
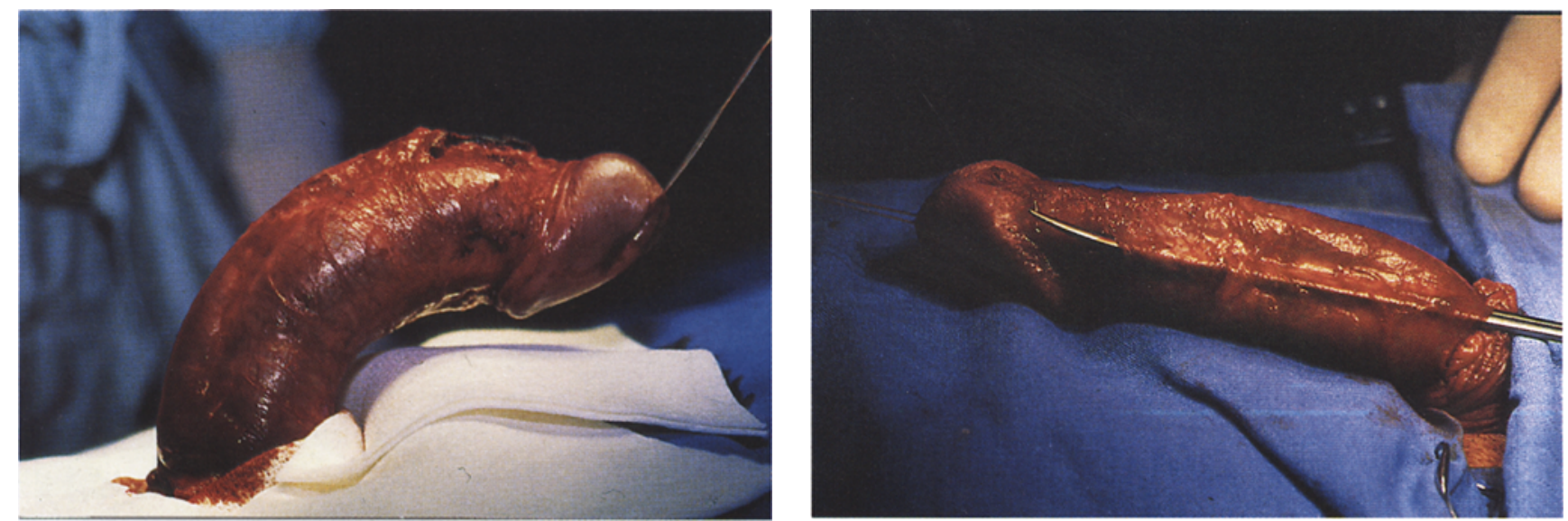

Figure 4: Exposition

Figure 5 : Dissection du fascia de buck isolement des pedicules dorsaux 

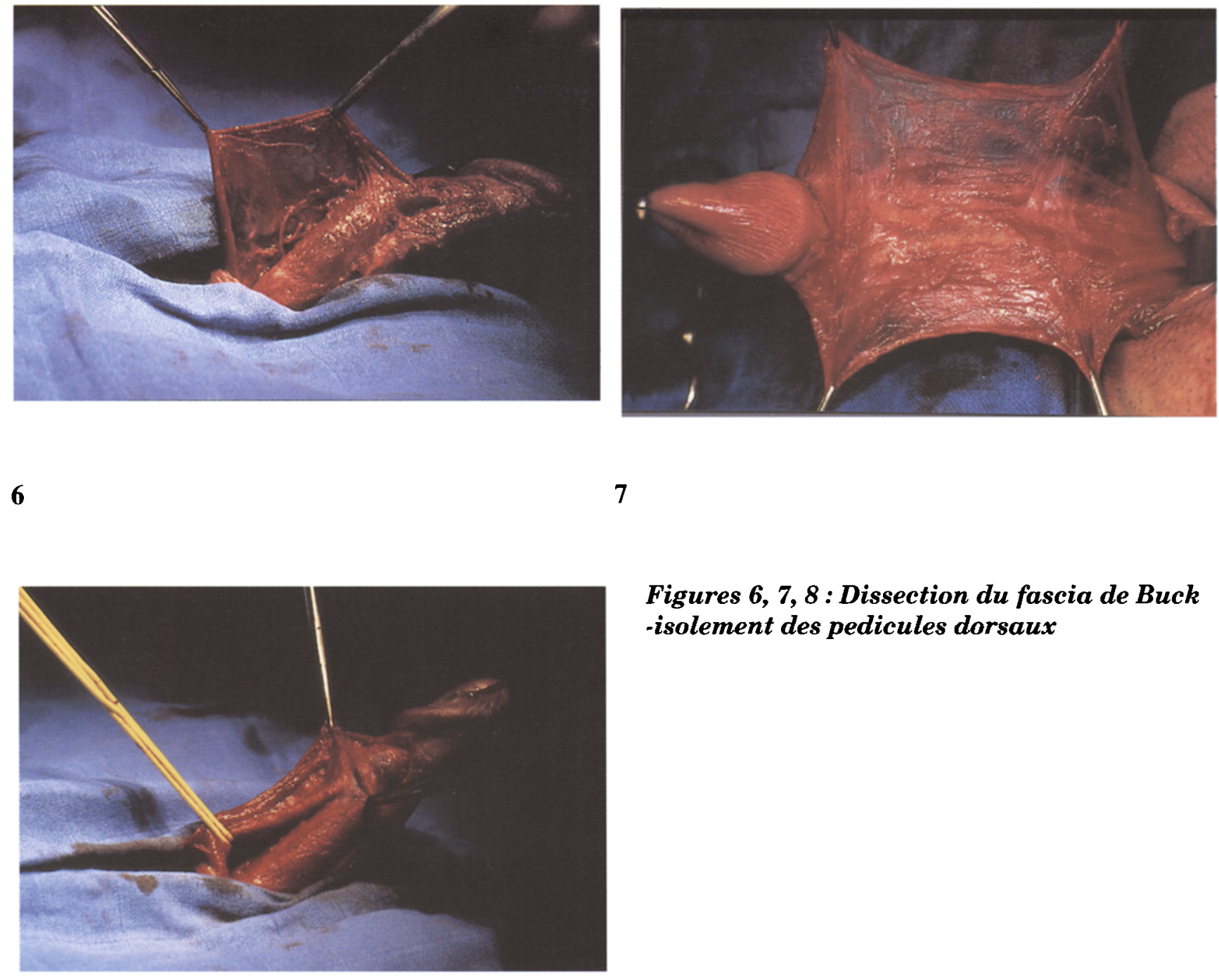

Figures 6, 7, 8 : Dissection du fascia de Buck -isolement des pedicules dorsaux

\section{8}

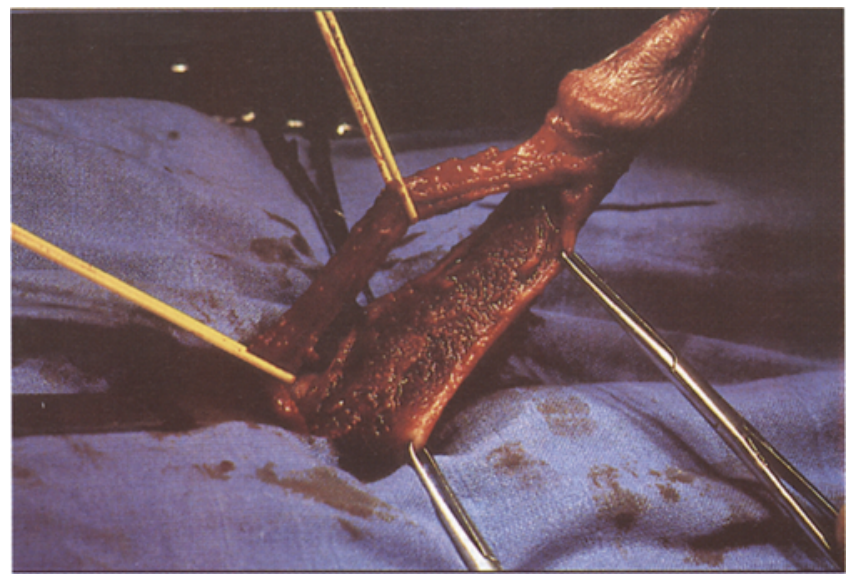

Figures 9 : Résection étendue des plaques et du septum

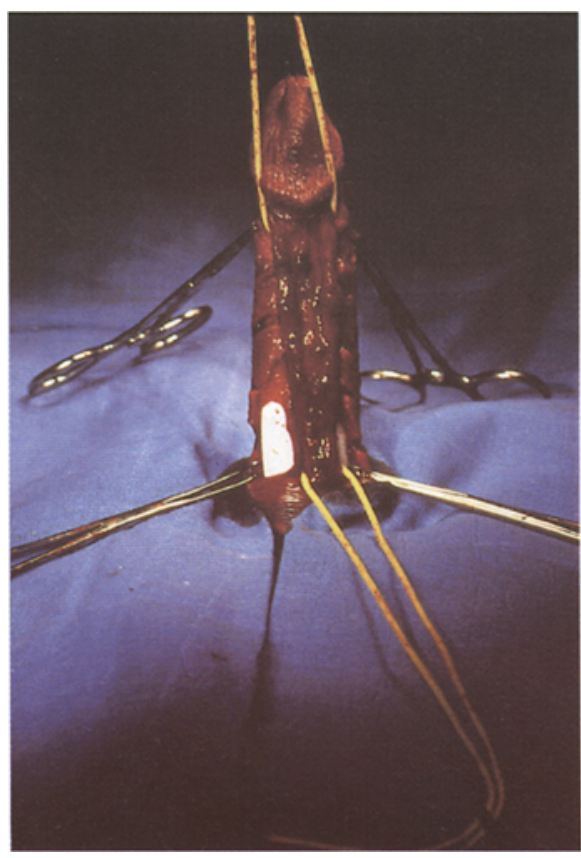

Figures 10 : Implants souples en place 


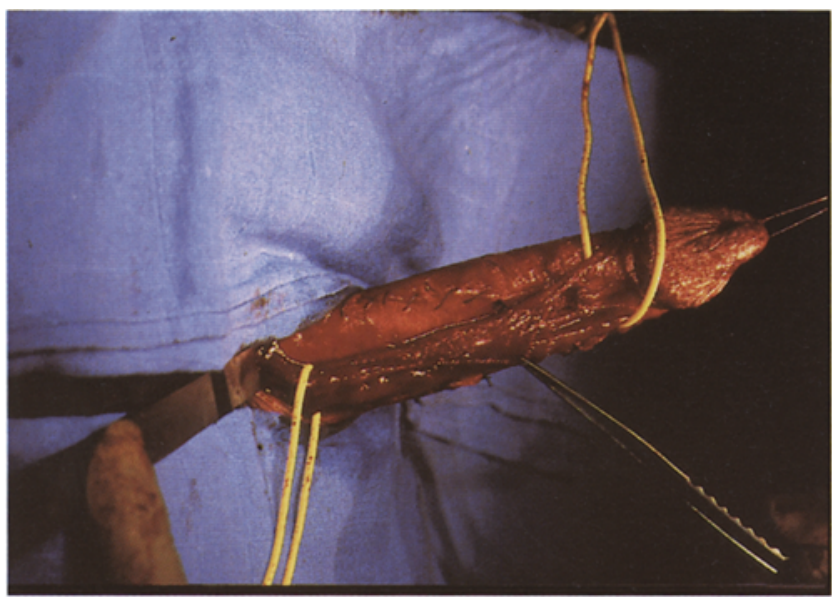

Figures 11 : Recouvrement par "produra»

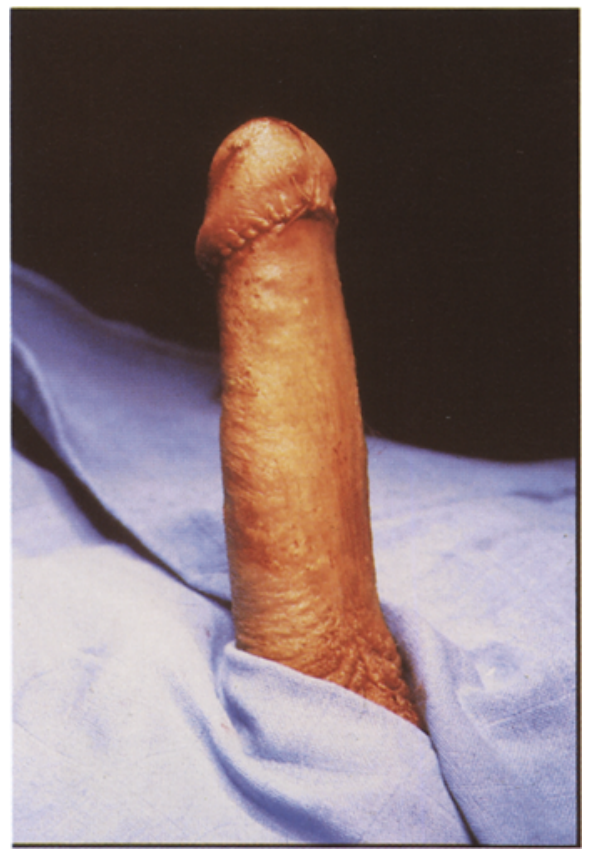

Figures 12 : Résultats

le premier but poursuivi. Il est atteint et donc la sexualité rétablie.

- Les désagréments liés à la nécessité d'exposer la face dorsale des corps caverneux en soulevant tous les éléments vasculo-nerveux sont, en majorité, réversibles dans les mois post-opératoires.

- L'érection complémentaire autour des implants est une réalité et demeure toujours possible.

- Il ne nous semble pas indispensable de recouvrir les zones excisées par une greffe, le Fascia de Buck paraissant avoir un rôle protecteur et hémostatique suffisant.

- La maladie de La Peyronie ne se reproduit pas après pose d'implants et les zones pathologiques respectées disparaissent, car la chéloïde peut se résorber spontanément lorsque l'on place un implant siliconé intracaverneux. Ces constatations très empiririques ont déjà été signalées (A. Leriche).

\section{CONCLUSION}

Nous pensons pouvoir tirer une impression positive de ces 22 observations de formes sévères de la maladie de La Peyronie, auxquelles on a osé s'attaquer, car elle sont souvent totalement abandonnées. Les interventions larges, délabrantes, téméraires qui ont été entreprises apportent une satisfaction d'ensemble fondée sur l'innocuité, et la qualité d'une majorité de résultats.

Les insatisfactions tiennent plus au contexte des couples pathologiques après l'intervention qu'aux résultats de la technique utilisée. On a rendu un comportement convenable 18 fois sur 22 à ceux qui n'avaient plus de vie sexuelle.

Ces opérations de résection très large de l'albuginée, du septum intercaverneux, complétées par la pose d'implants péniens sont très peu pratiquées en France et méritent d'être connues ; tant que la maladie de La Peyronie ne connaîtra que des palliatifs, on pourra y recourir dans des indications choisies et bien définies :

- la coudure extrême ;

- le racourcissement important ;

- l'impuissance.

Le choix des implants est un peu une affaire personnelle. Cependant, dans notre pratique l'orientation se fait vers les plus simples, les moins onéreux avec le désir d'un maniement aisé, et d'une tolérance éprouvée ; les implants souples paraissent réunir ces exigences. 


\begin{abstract}
Surgery of LaPeyronie's disease :
\end{abstract}

Our experience of austoni's procedure

M.ETCHeVERY, J.TIGNOL

Advanced stages of La Peyronie's disease, with severe recurvation are defined, for which radical surgery is necessary ; some well-known techniques are re-called, especially the famous AUSTONI's procedure.

A simplification of this wonderfull operation is possible and described, after large plaque removal and prosthesis implantation, no graft seems indispensable. Bücks Fascia, containing the dorsal nervous -vascular bundle, often thick, infiltrated by sclerosis, and resistant, is able to cover perfectly the defect. In addition was sometimes used a biologic artificial absorbable membrane.

22 cases are reported and the results after 12 to 60 months carefully studied. A majority are satisfied : length of the penis, sensibility of the glands, additionnal erections around implants are noted. Sexual intercourse begin again after 60 days, and 120 days are usually sufficient to reach the best.

Key words : La Peyronie's disease, Plaque removal, Penile implants 